\title{
Effects of Aerosols on Cloud Albedo: Evaluation of Twomey's Parameterization of Cloud Susceptibility Using Measurements of Ship Tracks
}

\author{
ANDREW S. ACKERMAN \\ NASA Ames Research Center, Moffett Field, California \\ OWEn B. TOON \\ Laboratory for Atmospheric and Space Physics and Program in Atmospheric and Oceanic Sciences, University of Colorado, Boulder, \\ Boulder, Colorado \\ Jonathan P. TAYLOR AND Doug W. Johnson \\ Meteorological Research Flight, The Met. Office, Farnborough, Hampshire, United Kingdom \\ Peter V. Hobbs and Ronald J. FereK* \\ Atmospheric Sciences, University of Washington, Seattle, Washington
}

(Manuscript received 1 August 1996, in final form 4 April 1997)

\begin{abstract}
Airborne measurements from the Meteorological Research Flight's Hercules C-130 and the University of Washington's Convair C-131A during the Monterey Area Ship Track field project are used to evaluate Twomey's analytic expression for cloud susceptibility, which describes the sensitivity of cloud albedo to changes in droplet concentrations. This expression incorporates assumptions about cloud physics, such as the independence of the cloud liquid water content and the width of the droplet size distribution on droplet concentrations. Averaged over all 69 ship track penetrations, cloud liquid water content decreased slightly and the droplet size distributions broadened from the ambient values. For the 17 cases for which albedos were measured during overflights, Twomey's parameterization represents the trend of albedo changes with droplet concentrations remarkably well, passing through the midpoints of the considerable spread in the data. The fortuitous agreement results from compensating changes in cloud properties. Together with the albedo changes, the changes in cloud liquid water content and droplet size distributions imply that cloud thickness usually increased in the ship tracks. Such an increase was observed on the occasions that changes in cloud thickness were recorded (in the Sanko Peace ship track during very clean ambient conditions). Unfortunately systematic measurements of cloud thickness were not made for most of the ship tracks observed. The greatest outlier in the data corresponds to measurements made under horizontally inhomogeneous ambient conditions; possible explanations for its divergence include an increase in cloud thickness or an error in matching above-cloud albedo measurements with in-cloud microphysics measurements.
\end{abstract}

\section{Introduction}

Low-lying stratiform clouds cover a third of the ocean surface and play an important role in the earth's radiative heat balance (Warren et al. 1988). Because they reflect more sunlight than the underlying ocean surface but emit thermal radiation at about the same temperature as

* Current affiliation: Office of Naval Research, Washington, D.C.

Corresponding author address: Dr. Andrew S. Ackerman, NASA Ames Research Center, MS 245-4, Moffett Field, CA 94035.

E-mail: ack@sky.arc.nasa.gov the surface, they cool the planet. Increased aerosol concentrations can produce higher droplet concentrations and thereby enhance cloud albedo (Twomey 1974). Higher droplet concentrations may also produce more persistent clouds with enhanced liquid water contents due to the suppression of drizzle in clouds with smaller droplets (Albrecht 1989). [In contrast, the theoretical results of Ackerman et al. (1995) suggest that cloud water can be depressed during the daytime when vertical mixing is suppressed.] Because pollution is a significant source of atmospheric aerosol, some of the global warming expected from carbon dioxide emissions may be offset by increases in the albedo of clouds downwind of pollution sources. A striking example of the effects 
of increased aerosol concentration on cloud albedo is provided by "ship tracks," which are long-lived, linear regions of enhanced cloud reflectivity sometimes observed in satellite imagery downwind of ships (Conover 1966; Coakley et al. 1987). During the Monterey Area Ship Track (MAST) field project off the coast of California during June 1994 a large number of ship tracks were sampled from instrumented aircraft and other platforms. Here we use airborne measurements to evaluate parameterizations linking aerosol pollution to the microphysical and optical properties of marine stratiform clouds.

The evaluation of the impact of pollution on global climate through the effects of aerosols on cloud albedo (the so-called indirect effect of aerosols on climate) in large-scale models requires realistic parameterizations of the relationships between aerosol concentrations and cloud properties. The aerosol-cloud relationships can be broken down into two parts: 1) the effects of aerosol emissions on cloud droplet concentrations, and 2) the effects of variations in cloud droplet concentrations on cloud albedo. The first link is affected by the chemical properties of the aerosol. Hobbs et al. (2000) and Durkee et al. (2000) have investigated the first link, using measurements of cloud condensation nuclei (CCN) in ship exhaust plumes and in the ambient atmosphere. The formation of ship tracks integrates both links; Coakley et al. (2000) have investigated the environmental conditions associated with formation of ship tracks (as seen in near-infrared satellite imagery) and found that ship track formation requires shallow boundary layers. In a shallow boundary layer the emitted aerosols are less diluted (than in deeper boundary layers) before they interact with clouds capping the boundary layer; hence the formation of ship tracks is more sensitive to the first link. Here we focus on the second link, namely, the effects of changes in cloud droplet concentrations on cloud albedo. Taylor et al. (2000) have also used the MAST observations to investigate this link. In this paper, we evaluate the validity of Twomey's (1991) analytic expression for the susceptibility of cloud albedo to changes in droplet concentrations.

Ship tracks are most prominent in satellite imagery at near-infrared wavelengths, where cloud droplets absorb solar energy (e.g., Coakley et al. 1987; Platnick et al. 2000). However, because the spectrum of solar energy incident on clouds is dominated by visible wavelengths, it is the reflection of sunlight at visible wavelengths that is relevant to the indirect effect of aerosols on climate. Consistent with Twomey's (1991) definition of cloud susceptibility, this paper addresses cloud albedos at visible wavelengths only.

The aerosols in ship plumes can overwhelm the ambient CCN concentrations by many orders of magnitude, representing the greatest direct impact of ship emissions on the atmosphere. Ships also emit water vapor and heat in their exhaust. The resulting buoyancy can accelerate the delivery of the aerosols to an overlying cloud layer.
Only once during the month-long experiment was cloud formation associated with a ship plume; on that occasion a line of cumulus clouds was observed (from the Naval Research Laboratory airship) to extend kilometers downwind in clear air adjacent to a low stratus deck (Frick and Hoppel 2000). However, on no other occasions were detectable perturbations of heat and moisture measured in the ship tracks (Hobbs et al. 2000; Frick and Hoppel 2000; Durkee et al. 2000).

In this paper we first briefly describe the measurements from MAST that we use in our analysis. Then we present a derivation of Twomey's analytic expression for cloud susceptibility in order to demonstrate the significance of the assumptions implicit in the expression. This is followed by evaluations of those assumptions and Twomey's (1991) analytic expression using the MAST measurements.

\section{Description of dataset}

Data from several sources are used in this study. Measurements of ship tracks produced by nine ships were made during six flights of the Meteorological Research Flight (MRF) C-130 Hercules aircraft. A brief description of the measurements is given by Taylor et al. (2000); a more complete description of the standard meteorological instrumentation is given by Rogers et al. (1995), of the cloud physics instrumentation by Martin et al. (1994) and Brown (1993), and of the radiation instrumentation by Kilsby et al. (1992) and Saunders et al. (1992). Droplet size distributions were measured with a Particle Measuring Systems (PMS) FSSP-100 (Forward Scattering Spectrometer Probe), which counted droplets between 1 - and 23.5 - $\mu \mathrm{m}$ radius, and a PMS 2D-C probe, which counted droplets between 12.5- and $200-\mu \mathrm{m}$ radius. Depth-of-field corrections were applied by MRF to the 2D-C measurements (Knollenberg 1970). For the radii where the two probes overlapped, we use arithmetic averages.

For each penetration by the MRF C-130 of a ship track from an identified vessel, ambient cloud properties are averaged over a few kilometers of flight path on either side of the ship track, and ship track properties are averaged over the duration of the penetration (subjectively identified as a region of enhanced cloud droplet concentrations).

Visible albedos (over the wavelength range of $0.3-$ $0.7 \mu \mathrm{m}$ ) above cloud tops were measured from the MRF C-130 with two pairs of Eppley pyranometers and then were similarly averaged to obtain ambient and ship track values. The albedo measurements were typically taken at heights ranging from $\sim 150$ to $\sim 350 \mathrm{~m}$ above cloud top. Although the instruments have a hemispherical field of view, $70 \%$ of the collected energy comes from a cone with a half-angle of $60^{\circ}$. Hence, for a height of $150 \mathrm{~m}$ above cloud top, the "footprint" of a measurement of 1-s duration is an oval $\sim 300 \mathrm{~m}$ wide and $\sim 400$ $\mathrm{m}$ long (stretched along the direction of flight at the 


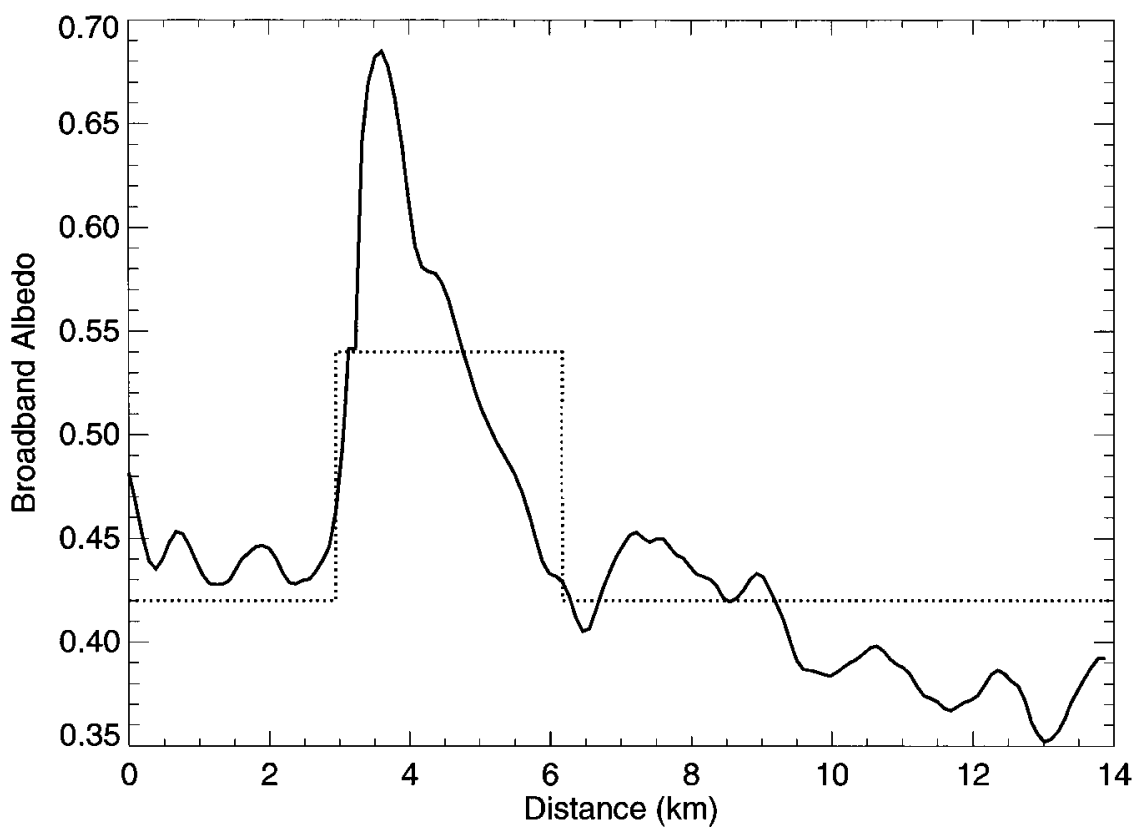

FIG. 1. Measured visible albedo (over the wavelength range of 0.3-3 $\mu \mathrm{m}$ ) obtained from the MRF C-130 (at a height $200 \mathrm{~m}$ above cloud top) over the ship track produced by the Hanjin Barcelona, plotted against distance (at an airspeed of $100 \mathrm{~m} \mathrm{~s}^{-1}$ ). Solid line is data recorded at $1 \mathrm{~Hz}$; dotted lines are averaged ambient and ship track values.

airspeed of $\sim 100 \mathrm{~m} \mathrm{~s}^{-1}$ ). Hence, an overflight of a kilometers-wide ship track (perpendicular to its axis) can yield a prominent, identifiable signal (Fig. 1). In regions with multiple ship tracks, navigation from ships' courses and measured wind velocities was used to identify the ships with their tracks.

We also investigate ship tracks produced by six ships using measurements made during six flights by the University of Washington (UW) Convair C-131A aircraft. A brief description of the instrumentation is given by Hobbs et al. (2000); a more detailed description is given by Hobbs et al. (1991). Droplet size distributions were measured with the same types of probes and using the same size-range settings as used by the MRF. However, another algorithm for depth-of-field corrections (Baumgardner 1987) was applied by UW to the 2D-C measurement. This correction resulted in a $2 \mathrm{D}-\mathrm{C}$ droplet size range between $50-$ and $212.5-\mu \mathrm{m}$ radius. Given the same droplet size distributions as input, the UW correction would result in a smaller total concentration of droplets and liquid water content in the 2D-C size range compared to the MRF correction.

For each penetration by the UW C-131A of a ship track from an identified vessel, ambient cloud properties were averaged over a few kilometers of flight on either side of the ship track, and ship track properties were averaged over the duration of the penetration. The edge of each ship track was identified as the location where the enhancement of particle concentrations (measured with a TSI CN model 3760) fell to $e^{-1}$ of the maximum enhancement during the penetration.

\section{Relationships between cloud droplet concentrations and cloud properties}

The sensitivity of cloud albedo $A$ to cloud droplet concentrations $N$ can be represented through cloud susceptibility, $d A / d N$ (Twomey 1991). For a given change in $N$, the cloud susceptibility can be used to predict the change in albedo: $\Delta A=d A / d N \times \Delta N$ (as discussed below, such a simplified application of the susceptibility is valid only for $\Delta N / N \ll 1$ ).

Although cloud susceptibility was defined by Twomey (1991) under the assumption that cloud water is independent of $N$, Platnick and Twomey (1994) extended the original definition of susceptibility to allow the possible dependence of cloud water on $N$. Here we consider the extended version of cloud susceptibility and evaluate some of the components on which it depends. The following derivation of cloud susceptibility borrows heavily from the presentation of Platnick and Twomey (1994).

By restricting the radiative wavelengths to the broadband visible range $(0.3-0.7 \mu \mathrm{m})$ and considering only cloud droplets and not aerosols, one can treat the scattering as conservative (no absorption) and the asymmetry parameter as a constant. These assumptions allow the cloud susceptibility to be expressed as

$$
\frac{d A}{d N}=\frac{d A}{d \tau} \frac{d \tau}{d N},
$$

where $\tau$ is the optical depth of the cloud. For droplets much larger than the wavelength of light (which therefore scatter in the geometric limit), 


$$
\tau=2 \pi \overline{r^{2}} N h
$$

where $\overline{r^{2}}$ is the mean square radius of the droplet size distribution and $h$ is the thickness of the cloud layer (it is assumed that the cloud droplet size distribution is independent of height). To cast (2) in terms of parameters that are more typically reported, we use the effective radius $r_{e}=\overline{r^{3}} / \frac{r^{2}}{}$, the liquid water content $L=$ (4/3) $\pi \rho_{w} N \overline{r^{3}}$, and Martin et al.'s (1994) radius parameterization $\overline{r^{3}}=k r_{e}^{3}$, resulting in

$$
\tau=2 \pi\left(\frac{3 L}{4 \pi \rho_{w}}\right)^{2 / 3}(k N)^{1 / 3} h
$$

Hence, the rightmost derivative in (1) may be evaluated from

$$
\frac{d \tau}{d N}=\frac{\tau}{3 N}\left(1+2 \frac{d(\ln L)}{d(\ln N)}+\frac{d(\ln k)}{d(\ln N)}+3 \frac{d(\ln h)}{d(\ln N)}\right) .
$$

It is often assumed that the dependencies of $L, k$, and $h$ on $N$ are insignificant (we will hereafter refer to these as the "cloud physics assumptions"), in which case

$$
\frac{d \tau}{d N}=\frac{\tau}{3 N}
$$

The first derivative on the right side of (1) can be evaluated from the analytic two-stream approximation (Bohren 1987):

$$
A \approx(1-g) \tau /[2+(1-g) \tau],
$$

where $g$ is the asymmetry factor. This relationship assumes horizontally homogeneous conditions, a surface albedo of zero, and that incident sunlight is spread uniformly over the downward hemisphere. Taking the derivative of (6) yields

$$
\frac{d A}{d \tau}=\frac{A(1-A)}{\tau} .
$$

Together with (5) and all the preceding assumptions, this yields a simple analytic expression for susceptibility:

$$
\frac{d A}{d N}=\frac{A(1-A)}{3 N} .
$$

Below we evaluate the validity of (8). Breaking it down into the product of two factors as in (1), we will first evaluate the dependencies of $L, k$, and $h$ on $N$ in (4) and then evaluate (8). But first we compare and contrast the datasets from the two aircraft.

The two aircraft (MRF C-130 and UW C-131A) have different operating ranges and complements of instruments and flew missions that were rarely in the same area simultaneously. The average conditions measured by each aircraft are summarized in Table 1. The MRF measurements were obtained at significantly lower droplet concentrations on average than were the UW measurements. The other systematic differences between the
TABLE 1. Average ambient and ship track conditions measured by the two aircraft. Here, $N$ is total droplet number concentration, $L$ is liquid water content, $k$ is defined through the relation $\overline{r^{3}}=k r_{\mathrm{e}}^{3}$, and $r_{e}$ is the effective radius of the droplet size distribution. The averages for the combined dataset are weighted by the number of individual cases (29 for the MRF dataset and 40 for the UW dataset).

\begin{tabular}{cccc}
\hline \hline & \multicolumn{3}{c}{ Dataset } \\
\cline { 2 - 4 } Parameter & MRF & UW & Combined \\
\hline$N\left(\mathrm{~cm}^{-3}\right)$ & & & \\
Ambient & 52 & 94 & 76 \\
Ship track & 98 & 162 & 135 \\
$L\left(\mathrm{~g} \mathrm{~m}^{-3}\right)$ & & 0.21 & 0.19 \\
Ambient & 0.17 & 0.21 & 0.18 \\
Ship track & 0.15 & 0.75 & 0.64 \\
$k$ Ambient & 0.49 & 0.63 & 0.57 \\
Ship track & 0.49 & & 12 \\
$r_{e}(\mu \mathrm{m})$ & & 10 & 9 \\
Ambient & 15 & 8 & \\
Ship track & 10 & & \\
\hline
\end{tabular}

two datasets (discussed further below) may result from the differences in average droplet concentrations and/ or the differences in the instrumentation (and data processing) on the two aircraft.

The first term of interest in (4) is the dependence of $L$ on $N$. Radke et al. (1989) reported a significant enhancement of cloud water from in situ measurements of a pair of ship tracks. They attributed the enhancement to a suppression of precipitation due to the reduction in droplet size in the ship tracks (Albrecht 1989). Platnick and Twomey (1994) showed that such an enhancement corresponded to an increase of $80 \%$ in susceptibility [i.e., $2 d(\ln L) / d(\ln N)=0.8$ in (4)] over the assumption that cloud water is independent of $N$. However, Leaitch et al. (1992) found no dependence of cloud water on droplet concentration in their extensive measurements of low-lying continental clouds (in which droplet concentrations were generally between 80 and $400 \mathrm{~cm}^{-3}$ ). In a modeling study of ship tracks, Ackerman et al. (1995) found that drizzle suppression resulted in an enhancement of cloud water through the upper regions of their simulated ship tracks (but that it was depressed in the lower reaches of the simulated ship tracks). However, when solar heating in the cloud layer significantly reduced vertical mixing, the region of enhanced cloud water became so thin that the cloud water was depressed throughout most of the depth of the ship track (see also Ferek et al. 2000).

The changes in liquid water content (between ambient cloud samples and the ship tracks) as a function of the changes in $N$ measured during MAST are shown in Fig. 2. Amidst the considerable scatter in the data, the overall trend is a reduction of liquid water content in the ship tracks, with a greater reduction measured by MRF. As seen in Table 2 , the mean value of $\Delta(\ln L) / \Delta(\ln N)$ for the combined dataset is -0.17 , with a standard deviation of 0.57 . This mean value corresponds to an expected reduction of susceptibility of $34 \% \pm 114 \%$ from the 

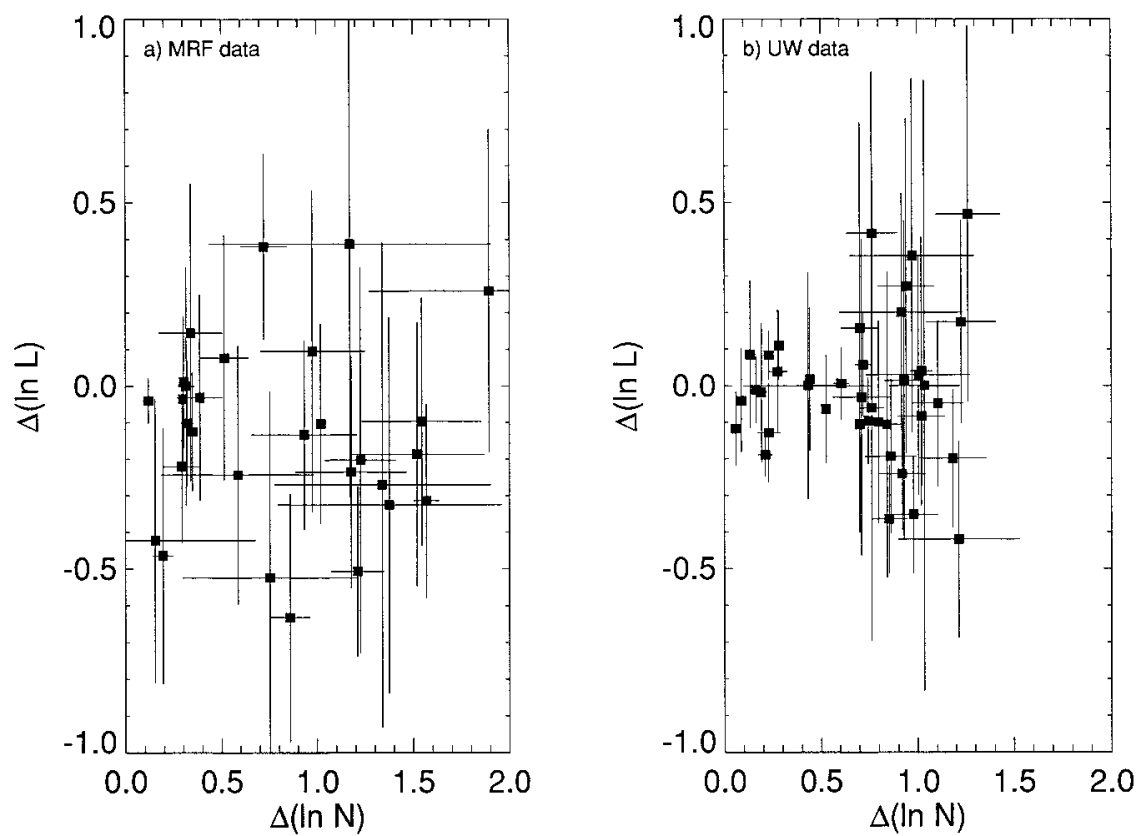

FIG. 2. Measured changes in liquid water content $(L)$ plotted against the changes in cloud droplet concentration $(N)$ obtained from (a) the MRF C-130 and (b) the UW C-131A. For each data point, the value plotted is $\ln$ (ship track value) - $\ln$ (ambient value), where "ln" is the natural logarithm. The error bars are obtained from the variances of the ambient measurements only.

assumption in (5) that cloud water is independent of $N$. Although this reduction is significant, its uncertainty is very great. A linear least squares fit through the combined data (and forced through the origin, since no change in liquid water should result from no change in droplet concentration) results in a slope of -0.083 and a correlation coefficient of -0.24 , which is significant at the $99 \%$ level but does not indicate a strong dependence of $\Delta(\ln L)$ on $\Delta(\ln N)$. Notably, the average change is in the opposite sense of that in the only previous in situ measurements of ship tracks (Radke et al. 1989) and is consistent with the model predictions of Ackerman et al. (1995) for daytime conditions.

Martin et al. (1994) analyzed a large set of airborne measurements of stratocumulus clouds taken over the eastern Pacific, the South Atlantic, the subtropical North

TABLE 2. Average changes measured by the two aircraft. The change ( ) is defined as the ship track value minus the ambient value. The parameters are defined in Table 1.

\begin{tabular}{cccc}
\hline \hline & \multicolumn{3}{c}{ Dataset } \\
\cline { 2 - 4 } Quantity & MRF & UW & Combined \\
\hline M(ln $L) / \Delta(\ln N)$ & & -0.07 & -0.17 \\
Mean & -0.31 & 0.44 & 0.57 \\
Std dev & 0.70 & & -0.14 \\
$\Delta(\ln k) / \Delta(\ln N)$ & & 0.58 & 0.53 \\
Mean & -0.002 & & -0.34 \\
$\operatorname{Std}$ dev & 0.44 & -0.28 & 0.26 \\
$\Delta\left(\ln r_{e}\right) / \Delta(\ln N)$ & & 0.21 & \\
Mean & -0.44 & 0.29 &
\end{tabular}

Atlantic, and the North Sea. They found that droplet size distributions (measured with an FSSP only) of clouds in continental air masses were broader than those in maritime air masses. For their parameterization $\overline{r^{3}}=$ $\overline{k r_{e}^{3}}$, they measured average values for $k$ of 0.80 and 0.67 in maritime and continental air masses, respectively $[k$ $=1$ for a monodisperse droplet size distribution; for a lognormal size distribution with a geometric standard deviation $\sigma_{g}, k=\exp \left(-3 \ln ^{2} \sigma_{g}\right)$ (see Gerber 1996)]. In light of (4), this variation of $k$ between air masses would suggest that the term $d(\ln k) / d(\ln N)$ should decrease susceptibility below the assumption of a constant value of $k$.

Examples of droplet size distributions obtained during the MAST experiment are shown in Fig. 3. For the ambient conditions in the first case (Fig. 3a), the total droplet concentration was $71 \mathrm{~cm}^{-3}$ and the narrow number distribution $(k=0.8)$ was dominated by condensation droplets $(r<10 \mu \mathrm{m})$. The average droplet concentration increased to $243 \mathrm{~cm}^{-3}$ in the ship track, and the overall distribution broadened $(k=0.64)$, consistent with the measurements of Martin et al. (1994). The overall change in $k$ was dominated by the broadening of the distribution of condensation droplets; the concentrations of drizzle droplets $(r>10 \mu \mathrm{m})$ decreased, but this decrease did not result in an overall narrowing of the distribution.

For the second case (Fig. 3b) the ambient droplet concentrations were very low $\left(9 \mathrm{~cm}^{-3}\right)$ and the number distribution was broad $(k=0.23)$ and strongly bimodal, with the dominant peak due to drizzle droplets. In the 

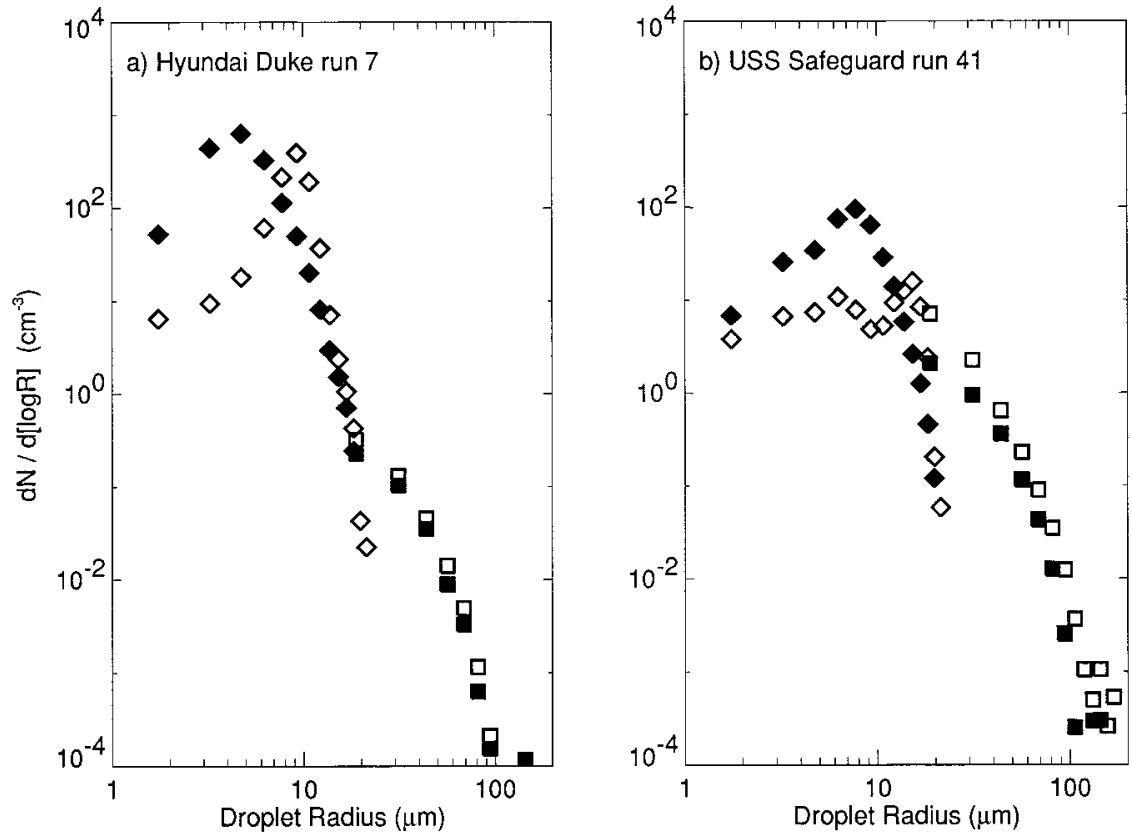

FIG. 3. Measured droplet size distributions obtained from the MRF C-130. Open symbols are for ambient conditions and closed symbols the ship tracks produced by (a) the Hyundai Duke and (b) the USS Safeguard. FSSP measurements are represented as diamonds, 2D-C measurements as squares; both are included in the instrument overlap region (12.5-25- $\mu \mathrm{m}$ radius). The ambient cloud was averaged over a total of $12 \mathrm{~km}$ of flight on both sides of each ship track.

ship track the average droplet concentration increased to $36 \mathrm{~cm}^{-3}$. In contrast with the first case (Fig. 3a), the overall distribution narrowed $(k=0.32$ in the ship track). This change is more in line with the "traditional" view of the expected contrast between maritime and continental droplet size distributions (Wallace and Hobbs 1977, pp. 168-169). In that view, droplet size distributions by number are broad under clean maritime conditions (low $N$ ) and are narrower and peaked at smaller sizes under continental conditions (high $N$ ).

The changes in $k$ measured during MAST are shown in Fig. 4 and summarized in Table 1. The average change measured by MRF is negligible, in contrast to the results from the broader survey of Martin et al. (1994). However, the droplet distributions obtained by Martin et al. (1994) were measured with an FSSP alone, while the MAST data were measured with an FSSP and a 2D-C probe together, which results in a greater sensitivity to drizzle droplets. If an increase in total droplet concentration were to result in a broadening of the mode of condensation droplets and a suppression of the drizzle mode, the FSSP alone would record a decrease in $k$, while the FSSP and 2D-C together could record an increase in $k$. This hypothesis is supported by analyzing the MRF measurements taken during MAST using the FSSP alone. In this case the average value of $k$ in the ambient clouds was 0.74 and fell to 0.66 in the ship tracks [more consistent with the results of Martin et al. (1994)]. But the combined (FSSP and 2D-C) data are more pertinent to the sensitivity of cloud albedo to changes in droplet concentrations, and the MRF data indicate that the third term on the right side of (4) can be ignored.

In contrast, the UW data indicate a decrease from an average $k=0.75$ in the ambient clouds to 0.63 in the ship tracks (Fig. 4b and Table 1). This change corresponds to a mean value of $\Delta(\ln k) / \Delta(\ln N)=-0.25$, with a standard deviation of 0.58 , corresponding to an expected reduction of susceptibility of $25 \% \pm 58 \%$ from the assumption of a constant value of $k$ in (5). A linear least squares fit through the UW data (forced through the origin) results in a slope of -0.24 and a correlation coefficient of 0.71 , which indicates a robust dependence of $\Delta(\ln k)$ on $\Delta(\ln N)$. The dilemma of contradictory results from the two aircraft is not easily resolved. The difference could be attributable to the different corrections used for the 2D-C measurements (an issue with no obvious resolution). Here we assume that the difference is due to the different microphysical regimes measured by the two aircraft (see Table 1) and average the two datasets together. As shown in Table 2, the combined dataset results in an expected reduction of the susceptibility of $14 \% \pm 53 \%$ from the assumption of a constant value of $k$ in (5). Alternatively, a least squares fit through the combined data yields a slope of -0.12 and a correlation coefficient of -0.32 .

Taken together, the averaged measurements of $L$ and $k$ for the combined dataset yield an expected reduction of the susceptibility of $48 \% \pm 126 \%$ from the cloud physics assumptions in (5). Although this represents a 

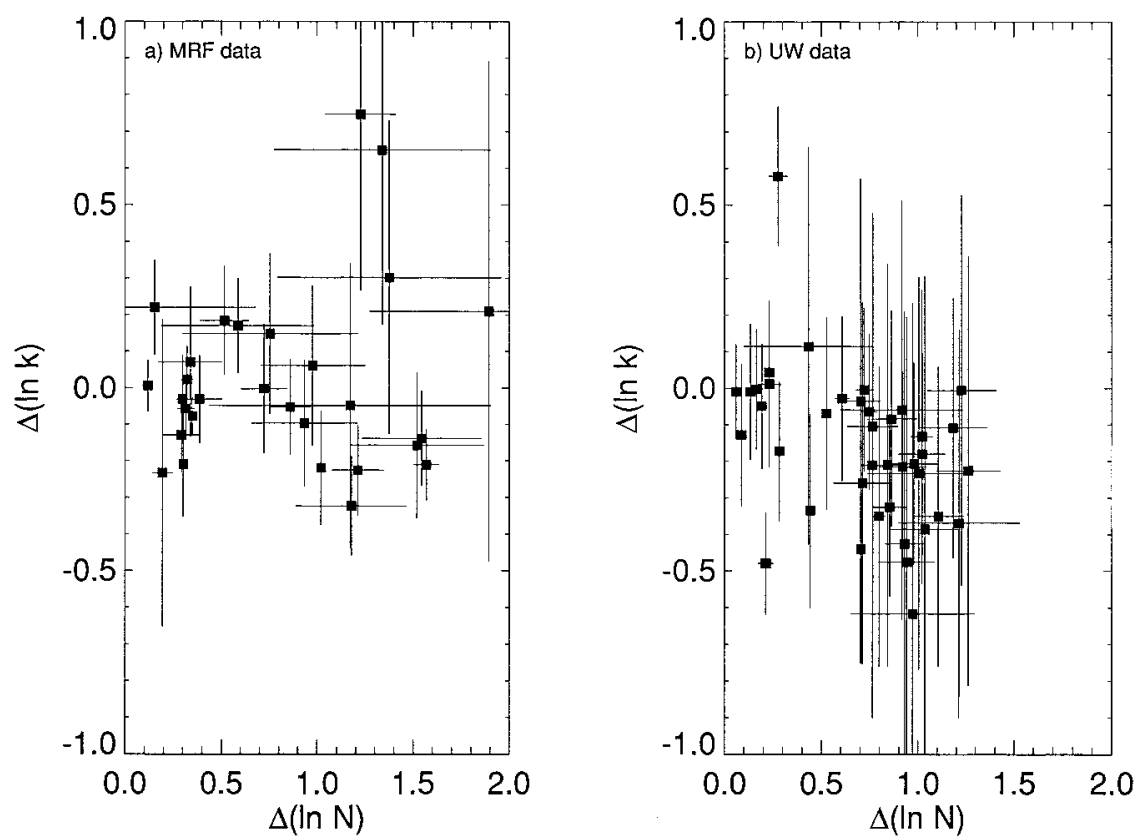

FIG. 4. Measured changes in $k$ (defined through the relation $\overline{r^{3}}=k r_{e}^{3}$ plotted against the changes in cloud droplet concentration $(N)$ from (a) the MRF C-130 and (b) the UW C-131A. Further details provided in the caption to Fig. 2. The errors for the UW data are overestimates because no covariances were available.

significant departure from the analytic expression (5), the uncertainty in the departure is substantial. Note that the measured changes in $L$ and $k$ reinforce each other in (4).

Another way to evaluate the assumptions that $L$ and $k$ are independent of $N$ is to examine the dependence of the droplet effective radius on droplet concentration. Taking the derivative of $L=(4 / 3) \pi \rho_{w} N \overline{r^{3}}$ yields

$$
\frac{d(\ln L)}{d(\ln N)}=1+\frac{d(\ln k)}{d(\ln N)}+3 \frac{d\left(\ln r_{e}\right)}{d(\ln N)} .
$$

If we assume that $L$ and $k$ are independent of $N$, the predicted relationship is

$$
\frac{d\left(\ln r_{e}\right)}{d(\ln N)}=-\frac{1}{3} .
$$

Figure 5a shows that relationship (10) fits the MRF measurements well: the least squares fit (forced through the origin), with a slope of -0.38 and a correlation coefficient of -0.95 , nearly overlies the analytic relationship (10). The least squares fit through the UW data (Fig. 5b), with a slope of -0.28 and correlation coefficient of -0.92 , falls on the other side of the analytic relationship (10). These strong correlations are reflected in the statistics of $\Delta\left(\ln r_{e} / \Delta(\ln N)\right.$ shown in Table 2, where the standard deviations are seen to be smaller than their mean values (a rare occurrence with this dataset). With respect to changes in the droplet effective radius in (9), the measured changes in $L$ and $k$ offset each other [in contrast to their reinforcement in (4)], resulting in an overall relationship that is well described by (10).

Cloud thickness $(h)$ is the final term in (4). Pincus and Baker (1994) used a mixed-layer model with parameterized microphysics and found $h$ and boundary layer thickness to be strongly dependent on $N$, because of the effect on the boundary layer energy budget of changes in the amount of precipitation reaching the surface. For instance, when $N$ doubled from 50 to $100 \mathrm{~cm}^{-3}$ in their simulations $h$ increased significantly, from 185 to $235 \mathrm{~m}$. This increase corresponds to $\Delta(\ln h) / \Delta(\ln N)$ $=0.35$, or a $105 \%$ enhancement of susceptibility according to our analysis. Although they found the enhancement to decrease with increasing $N$ (e.g., only a $55 \%$ enhancement when $N$ doubled from 200 to 400 $\mathrm{cm}^{-3}$ ), the hypothesized enhancement should be detectable for most of the MAST data.

Ackerman et al. (1993) used a turbulence closure model with explicit microphysics and found that when droplet concentrations fall to values $<10 \mathrm{~cm}^{-3}$ then a cloud-topped marine boundary layer can collapse to a shallow, surface-driven, fog layer. The predicted collapse occurs under conditions of low $N$ because the low droplet concentrations result in efficient drizzle production and hence a depletion of cloud water. The depleted cloud water causes the cloud layer to become so optically thin that it can no longer drive vertical mixing by cloud-top radiative cooling, thereby allowing the subsiding inversion air to compress the boundary layer. Ackerman et al. (1995) predicted that an injection of 

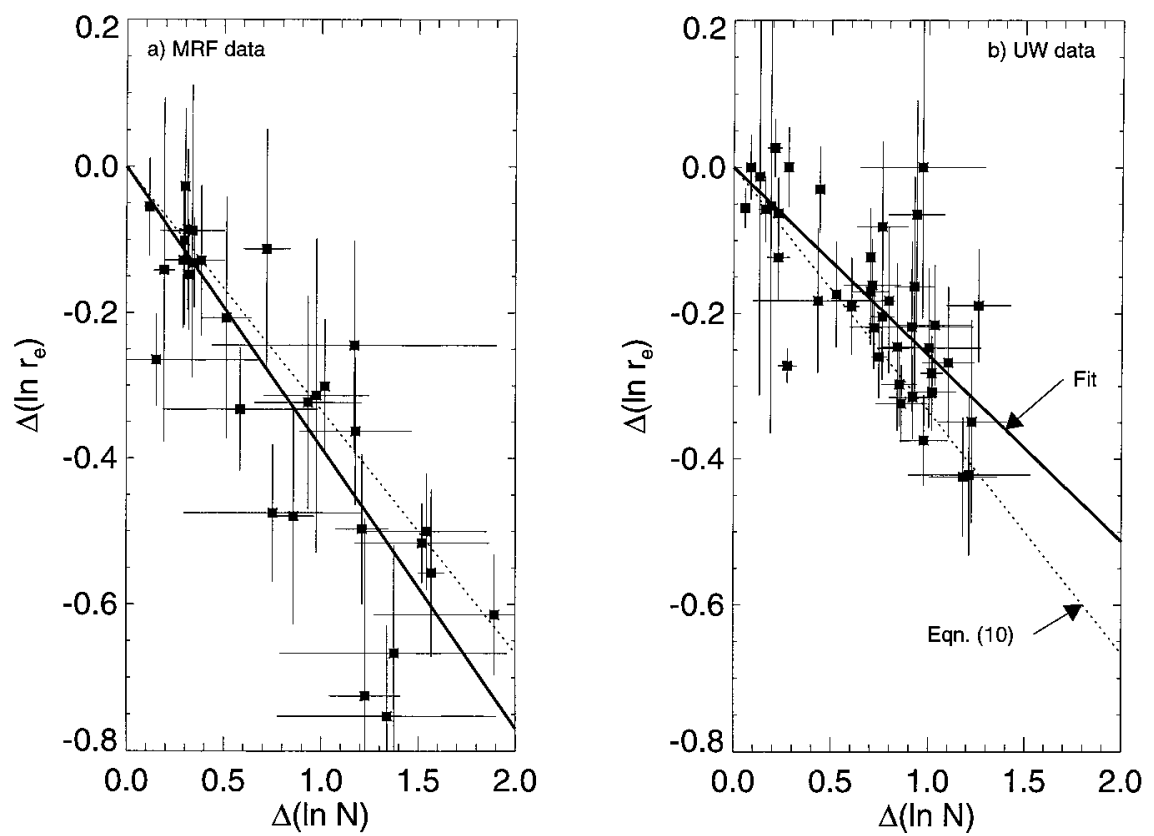

FIG. 5. Measured changes in droplet effective radius plotted against the changes in cloud droplet concentration $(N)$ from (a) the MRF C-130 and (b) the UW C-131A. The solid lines are least squares fits to the data (forced through the origin); dotted lines are the analytic relationship from (10). Further details provided in the caption to Fig. 2.

CCN (for instance, from a ship exhaust plume) into such a collapsed boundary layer could result in a reversal of the collapse, causing the cloud and boundary layer to deepen. Although this response of the boundary layer depth to changes in droplet concentrations is in the same sense as predicted by Pincus and Baker (1994), the two mechanisms are distinct (Pincus and Baker assumed a fixed radiative cooling rate). Notably, the ambient conditions for the ship track produced by the Sanko Peace were consistent with the state of the collapsed boundary layer as predicted by Ackerman et al. (1993), and also corresponded to the regime of maximum sensitivity of $h$ to $N$ according to the model of Pincus and Baker (1994). Consistent with both models' predictions, the boundary layer was observed to rapidly deepen by $\sim 100$ $\mathrm{m}$ in the Sanko Peace ship track. Hence, the dependence of $h$ on $N$ may be a term that should not be ignored in (4). The ship track produced by the Sanko Peace is the primary topic of a paper by Taylor and Ackerman (1999), so we will not discuss it further here. Unfortunately, except for this one case study, a direct assessment of the changes in the depths of the boundary layers and cloud layers is beyond the scope of the present dataset, since systematic measurements of $h$ together with $N$ were not made during the MAST field program.

Since we are unable to directly evaluate systematic changes in cloud thickness, we will next evaluate (8). We have shown that the first two cloud physics assumptions (that $L$ and $k$ are independent of $N$ ) do not appear to be valid in this dataset and that the measured changes are expected to reduce the susceptibility by $48 \%$ $\pm 126 \%$ from the analytic relationship (5). If cloud thickness did not change significantly in the ship tracks and if the radiative assumptions (e.g., those made in the two-stream derivation of cloud albedo) are valid, then Twomey's analytic expression for cloud susceptibility (8) should overpredict the observed susceptibilities by about a factor of 2. [Although Eq. (8) was derived under the assumption of horizontally homogeneous conditions, the ship tracks clearly cannot be described as such. We note that ship tracks are typically kilometers wide, but the footprint of the radiometers is typically $<500$ $\mathrm{m}$ across. Hence, the values analyzed here represent horizontal averages over horizontally inhomogeneous clouds, and any errors due to horizontal inhomogeneity are present in the data.]

It should be noted that there is some difficulty in comparing the analytic expression (8), which is a differential, with the measurements, which are finite differences. The explicit nonlinear dependence on $N$ can be treated by taking the derivative of $A$ with respect to the logarithm of $N$ [the independent axis we use is $\Delta(\ln N)]$. However, the nonlinearity of $A$ itself cannot be treated so simply in the analytic expression. Taylor et al. (2000) treat this difficulty by evaluating the analytic prediction iteratively: first they increment $N$ from its ambient value by a small $N$, then use the cloud physics assumptions to recalculate the cloud properties, then recalculate the susceptibility, and iterate this process until $N$ in the ship track is reached, with the final change of albedo taken from the sum of the incremental chang- 


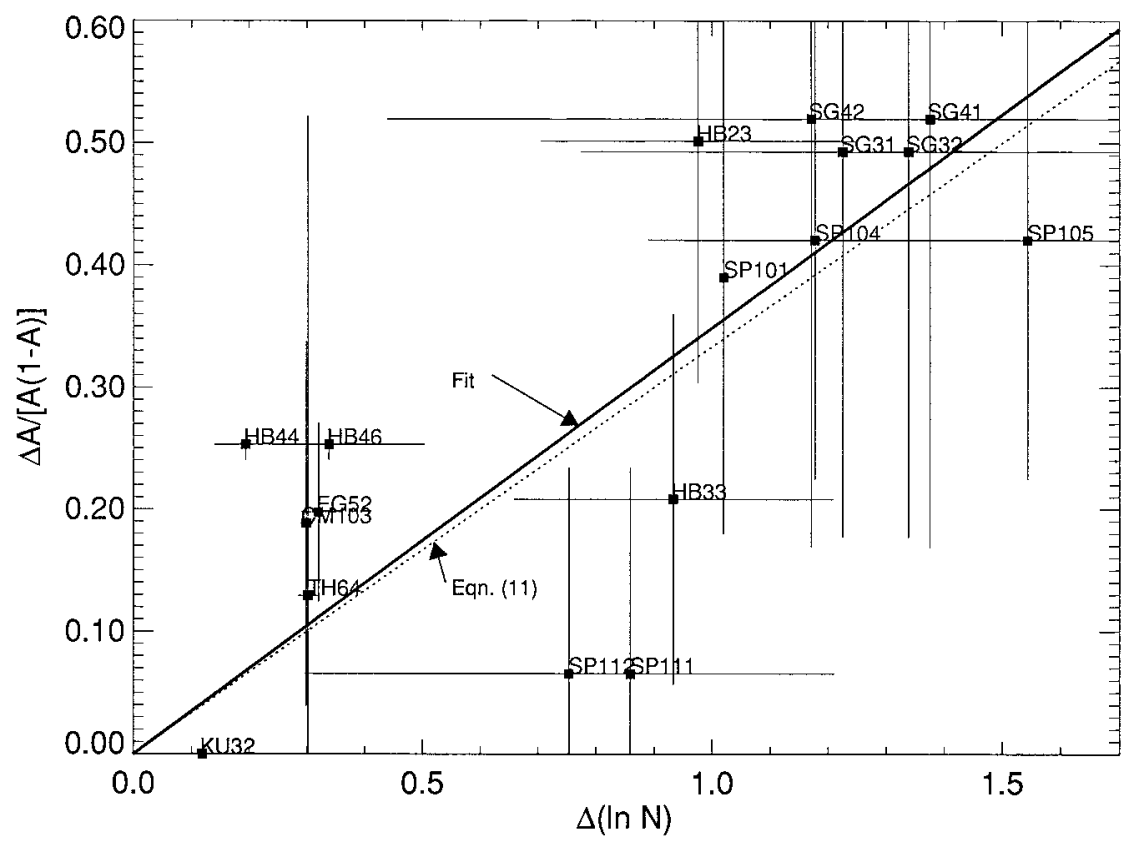

FIG. 6. Measured values of $\Delta A /(\bar{A}[1-\bar{A}])$ plotted against the changes in cloud droplet concentration $(N)$. Albedos measured above cloud tops were identified with microphysics measurements taken in the clouds for the MRF data only (Taylor et al. 2000): $\Delta A$ is the difference between the ship track albedo and the ambient value, and $\bar{A}$ is the arithemetic average of the two. The dotted line represents Twomey's (1991) analytic expression for cloud susceptibility, expressed in finite-difference form as $\Delta A /(\bar{A}[1-\bar{A}])=\Delta(\ln N) / 3$. The solid line is a least squares fit to the data (forced through the origin). The key to the labels on the data points is given in Table 3. Further details provided in the caption to Fig. 2.

es. To evaluate the analytic expression, we use a less sophisticated treatment, simply taking the arithmetic average $\bar{A}$ (of the ambient value and that in the ship track), which is a good approximation since $\Delta A<\bar{A}$ in all the measurements. Hence, we calculate the expected change in albedo from (8) as

$$
\Delta A=\bar{A}(1-\bar{A}) \Delta[\ln N] / 3 .
$$

Figure 6 presents a comparison between Twomey's analytic expression and the subset of MAST measurements (ship identifiers are listed in Table 3) for which albedo measurements were taken above cloud top (17

TABLE 3. Key to labels in Figs. 6-8. Each data point in the figures is labeled by an identifier. The first two letters identify the ship that produced the ship track in which the measurements were made, and is decoded below. The numerical suffix in each label describes the flight "run" (which is here multiplied by 10 to eliminate the decimal point).

\begin{tabular}{cl}
\hline \hline Ship identifier & \multicolumn{1}{c}{ Ship name } \\
\hline HD & Hyundai Duke \\
KU & Kurama \\
SG & USS Safeguard \\
SP & Sanko Peace \\
CM & Cape May \\
TH & Tai He \\
EG & Ever Genius \\
HB & Hanjin Barcelona \\
\hline
\end{tabular}

cases). Amid the considerable scatter in the measurements, the analytic expression splits the data remarkably well. The value of $\Delta A$ calculated from (11) is an approximation of the analytic expression, because it is derived from the susceptibility, which is a differential. An exact form can be derived from the two-stream albedo relationship with the same assumptions:

$$
\Delta A_{\text {exact }}=A(1-A)(\chi-1) /[A(\chi-1)+1],
$$

where $\chi^{3}=1+\Delta N / N$, and $N$ and $A$ are the ambient droplet concentration and albedo (Platnick and Twomey 1994). A comparison between the exact form (12) and the approximation (11) is shown in Fig. 7, which indicates that the approximation (11) is accurate for these data.

The overall agreement between Twomey's analytic relationship and the data in Fig. 6 would superficially seem to support the underlying assumptions. However, we have shown that the measured changes in cloud water and the width of the droplet size distributions are expected to result in a significant underprediction of the susceptibilities compared to Twomey's relationship: using the 17 cases with albedo measurements, the average value of $2 \Delta(\ln L) / \Delta(\ln N)+\Delta(\ln k) / \Delta(\ln N)$, which appears in (4), is -0.61 with a standard deviation of 1.29 , suggesting an expected $61 \%$ average reduction in the susceptibility. Instead, the analytic relationship of $\Delta A$ / 


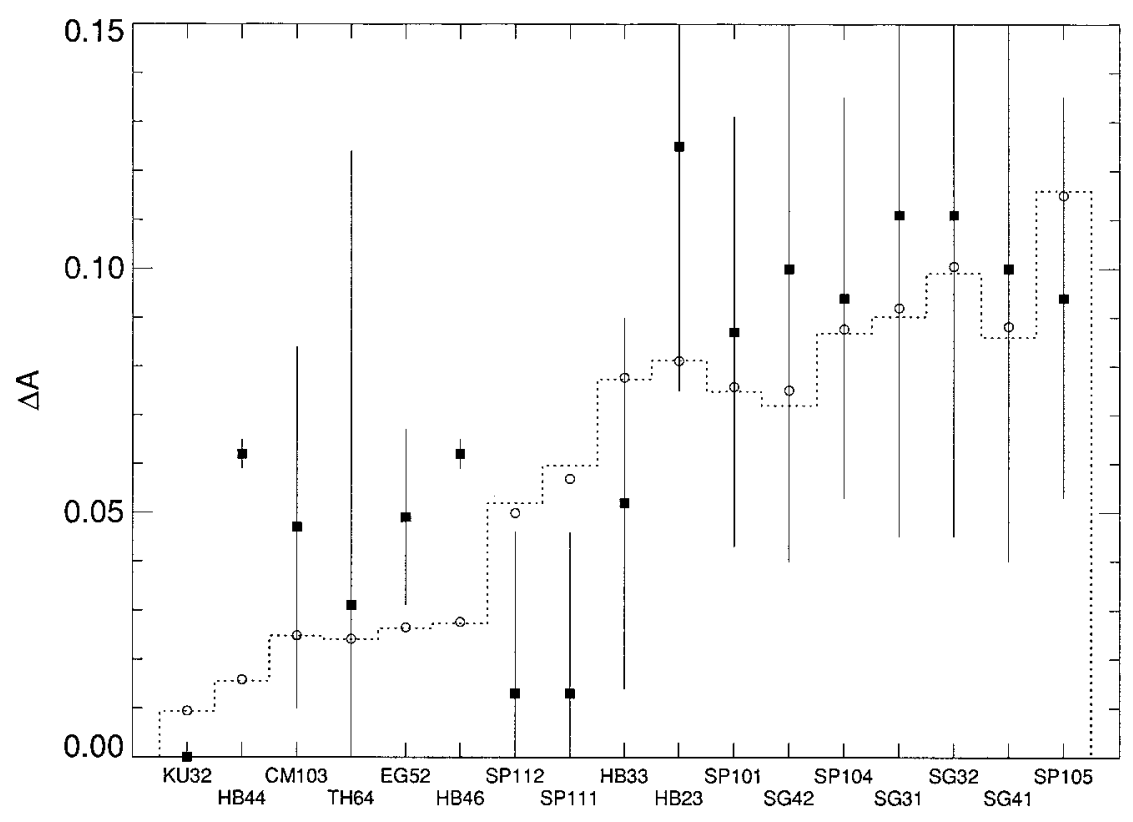

Ship track and aircraft flight run

FIG. 7. Measured values of $\Delta A$ plotted as solid squares with error bars, analytic values approximated from Eq. (11) plotted as open circles, and exact analytic values from Eq. (12) plotted with a dotted line. For comparison with the other figures, the data are sorted by $\Delta(\ln N)$. Further details provided in the caption to Fig. 6.

$[A(1-A) \Delta(\ln N)]=\frac{1}{3}$ is slightly exceeded by the measurements (Fig. 6): the average value of $\Delta A /[A(1-$ A) $\Delta(\ln N)]$ is 0.43 with a standard deviation of 0.30 , and the slope of a linear least squares fit (through the origin) of $\Delta A /[A(1-A)]$ as a function of $\Delta(\ln N)$ is 0.35 . A systematic increase of cloud thickness is therefore implied by our analysis; according to $(4), 3 \Delta(\ln h) / \Delta(\ln N)$ $>0.61$. For example, an average $15 \%$ increase in cloud thickness is implied for a doubling of droplet concentration. We can evaluate the implied increase in cloud thickness for each case by rearranging the finite-difference form of (4) and combining it with the two-stream albedo approximation:

$$
\begin{aligned}
\Delta(\ln h)= & \frac{\Delta A}{A(1-A)}-\frac{1}{3} \Delta(\ln N)-\frac{2}{3} \Delta(\ln L) \\
& -\frac{1}{3} \Delta(\ln k) .
\end{aligned}
$$

As shown in Fig. 8, (13) indicates an increase in cloud thickness for nearly all of the ship tracks. This result is supported by the observations in the ship track produced by the Sanko Peace, in which the cloud layer was observed to deepen significantly [by $\sim 100 \mathrm{~m}$ from a 270-m deep boundary layer; Taylor and Ackerman (1999)]. It is also consistent with the model results discussed above (Ackerman et al. 1995; Pincus and Baker 1994).

A notable outlier in Fig. 8 corresponds to the ship track produced by the Hanjin Barcelona (HB44), in which the implied value of $\Delta(\ln h)=0.58$ corresponds to a $79 \%$ increase in cloud thickness. Such an increase greatly exceeds the model predictions of Pincus and Baker (1994) at comparable droplet concentrations. These measurements were made in a decoupled boundary layer, with cumulus clouds rising into a patchy stratocumulus deck (Gasparovic 1995). Although the cloud water measured at flight level was significantly depressed in the ship track (from 0.31 to $0.17 \mathrm{~g} \mathrm{~kg}^{-1}$ ), it is possible that a thicker overlying stratocumulus cloud accounted for the enhanced albedo increase. Another possible explanation for the discrepancy also relates to the patchiness of the clouds on that day. The abovecloud albedo measurements were made at different times than the in situ measurements, and their spatial overlap with the in situ measurements is uncertain. The potential error in matching of the two types of measurements is greatest when the horizontal variations are greatest. Notably, the ambiguities in matching the two types of measurements resulted in the same above-cloud albedo measurements being used for another of the in situ measurements from the Hanjin Barcelona ship track (HB46). This data point is not an outlier in Fig. 8, suggesting that the discrepancy of HB44 is likely attributable to an error in matching the in-cloud microphysical measurements with the above-cloud albedo measurements. A possible further source of error under such conditions is the assumption of horizontal homogeneity implicit in the two-stream albedo approximation. 


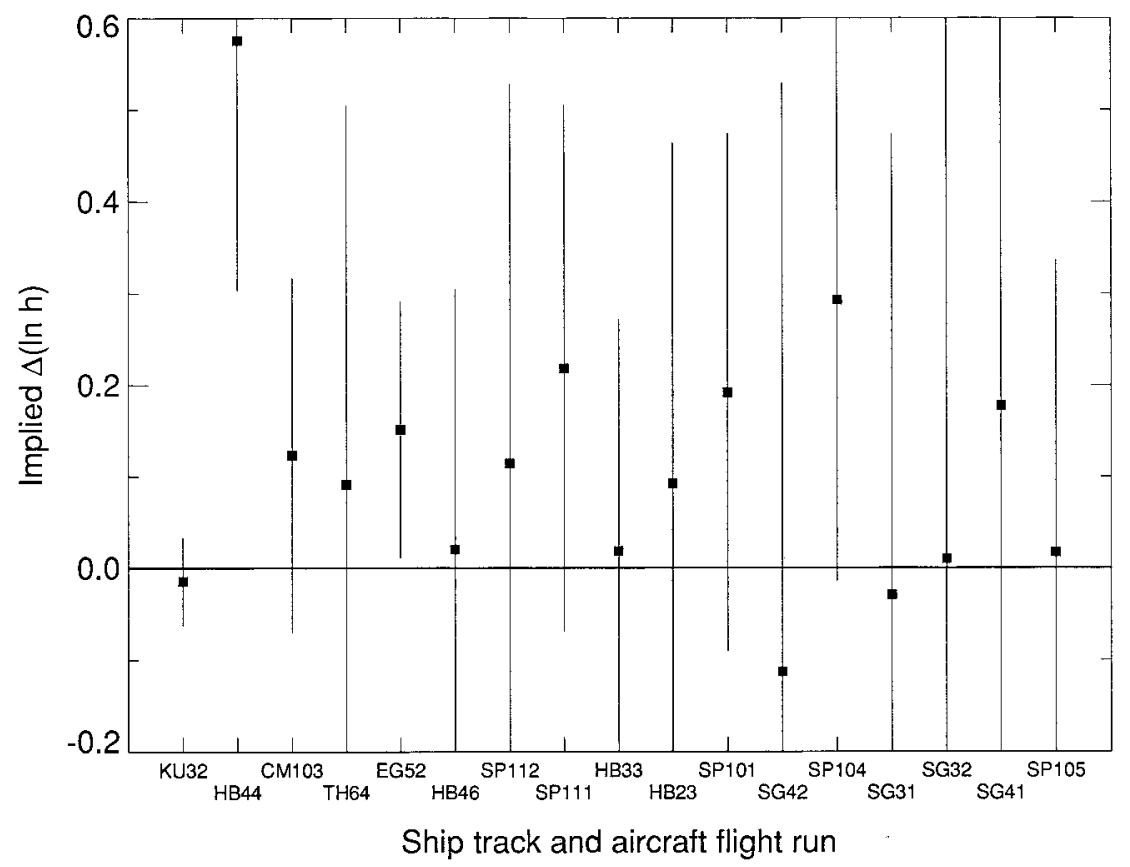

FIG. 8. Implied values of $\Delta(\ln h)$ from Eq. (13), in which $h$ is cloud physical thickness. Further details provided in the caption to Fig. 6 .

\section{Summary and conclusions}

We have used measurements from the Meteorological Research Flight's C-130 and the University of Washington's C-131A during the Monterey Area Ship Track (MAST) field project to evaluate Twomey's (1991) parameterization of the effects of changes in droplet concentrations on cloud albedo. We find that the parameterization generally describes the observed changes, with some caveats.

Twomey (1991) developed "cloud susceptibility" to be a sensitivity parameter describing changes in cloud albedo $A$ due to differential changes in cloud droplet concentrations $N$. In his analytic derivation of cloud susceptibility, he made several assumptions regarding the optical properties of cloud droplets and cloud layers, and further assumptions about cloud physics. Here we have focused on the cloud physics assumptions: namely, that cloud water, the width of the cloud droplet size distribution, and cloud thickness are all independent of $N$. (Regarding the second assumption, Twomey originally assumed a monodisperse size distribution, but we have shown here that Twomey's analytic relationship holds if the width of the size distribution, as parameterized by Martin et al. (1994), is independent of $N$.) The MAST in situ cloud measurements allowed us to examine the assumptions that cloud water and the width of the size distribution are independent of $N$. Averaged over all 69 ship track penetrations, cloud liquid water content decreased slightly and the droplet size distributions broadened. For the 17 cases in which albedos were measured during overflights, Twomey's parame- terization represents the trend of albedo changes with droplet concentrations remarkably well, passing through the midpoints of the considerable spread in the data. Combined with the decrease in cloud water and the broadening of the droplet size distributions, the susceptibility agreement implies that cloud thickness usually increased in the ship tracks. Such an increase was observed for the five measurements in which cloud thickness was reported [measurements made in the Sanko Peace ship track under very clean ambient conditions; Taylor and Ackerman (1999)], but systematic measurements of cloud thickness were not made during MAST.

The measurements in the Hanjin Barcelona's ship track represent an outlier of the dataset: a nearly $80 \%$ increase in cloud thickness is implied. It is unlikely that such a large change occurred. Those measurements were made under horizontally inhomogeneous conditions, which are likely to introduce errors when albedo measurements taken from above the cloud layer are matched to cloud microphysics measurements taken in the cloud layer at slightly different times and locations. The planeparallel assumptions in the analytic albedo expression are also violated under such conditions.

In conclusion, we have found that Twomey's (1991) analytic expression for cloud susceptibility generally represents the measurements well. Because it is such a simple formula, the agreement implies that changes in cloud albedo due to changes in cloud droplet concentrations can be predicted from very little information: namely, the ambient cloud albedo and droplet concentration, and the perturbed cloud droplet concentration. 
However, this agreement is fortuitous in these data due to offsetting effects from changes in several parameters (cloud liquid water content, the width of the droplet size distributions, and cloud thickness). Hence, some caution should be used employing Twomey's formulation, since the MAST data were collected under a limited range of meteorological conditions (predominantly shear-driven boundary layers $<1 \mathrm{~km}$ deep).

Acknowledgments. We would like to thank M. D. Glew of the U.K. Met. Office for processing the C-130 data and T. Garrett, S. Strader, and A. Rangno of the University of Washington for processing and providing the C-131A data. We also thank S. Platnick for helpful comments.

\section{REFERENCES}

Ackerman, A. S., O. B. Toon, and P. V. Hobbs, 1993: Dissipation of marine stratiform clouds and collapse of the marine boundary layer due to depletion of cloud condensation nuclei by clouds. Science, 262, 226-229.

,-- , and - , 1995: Numerical modeling of ship tracks produced by injections of cloud condensation nuclei into marine stratiform clouds. J. Geophys. Res., 100, 7121-7133.

Albrecht, B., 1989: Aerosols, cloud microphysics, and fractional cloudiness. Science, 245, 1227-1230.

Baumgardner, D., 1987: Corrections for the response time of particle measuring probes. Extended Abstracts, Sixth Symp. on Meteorological Observations and Instrumentation, New Orleans, LA, Amer. Meteor. Soc., 148-151.

Bohren, C. F., 1987: Multiple scattering of light and some of its observable consequences. Amer. J. Phys., 55, 524-533.

Brown, P. R. A., 1993: Measurements of the ice water content in cirrus using an evaporative technique. J. Atmos. Oceanic Technol., 10, 579-590.

Coakley, J. A., Jr., R. L. Bernstein, and P. A. Durkee, 1987: Effect of ship-stack effluents on cloud reflectivity. Science, 237, 10201022

— , and Coauthors, 2000: The appearance and disappearance of ship tracks on large spatial scales. J. Atmos. Sci., 57, 2765-2778.

Conover, J. H., 1966: Anomalous cloud lines. J. Atmos. Sci., 23, 778 785.

Durkee, P. A., and Coauthors, 2000: The impact of ship-produced aerosols on the microstructure and albedo of warm marine stratocumulus clouds: A test of MAST hypotheses 1i and 1ii. $J$. Atmos. Sci., 57, 2554-2569.

Ferek, R. J., and Coauthors, 2000: Drizzle suppression in ship tracks. J. Atmos. Sci., 57, 2707-2728.

Frick, G. M., and W. A. Hoppel, 2000: Airship measurements of ship's exhaust plumes and their effect on marine boundary layer clouds. J. Atmos. Sci., 57, 2625-2648.

Gasparovic, R. F., 1995: MAST experiment operations summary. S1R-95-07, Applied Physics Laboratory, The Johns Hopkins University, Laurel, MD, 300 pp. [Available from Applied Phys- ics Laboratory, The Johns Hopkins University, Johns Hopkins Road, Laurel, MD 20723.]

Gerber, H., 1996: Microphysics of marine stratocumulus clouds with two drizzle modes. J. Atmos. Sci., 53, 1649-1662.

Hobbs, P. V., L. F. Radke, J. H. Lyons, R. J. Ferek, D. J. Coffman, and T. J. Casadevall, 1991: Airborne measurements of particle and gas emissions from the 1990 volcanic eruptions of Mount Redoubt. J. Geophys. Res., 96, 18 735-18 752.

- and Coauthors, 2000: Emissions from ships with respect to their effects on clouds. J. Atmos. Sci., 57, 2570-2590.

Kilsby, C. G., D. P. Edwards, R. W. Saunders, and J. S. Foot, 1992: Water-vapour continuum absorption in the tropics: Aircraft measurements and model comparisons. Quart. J. Roy. Meteor. Soc., 118, 715-748.

Knollenberg, R. G., 1970: The optical array: An alternative to scattering or extinction for airborne particle size detection. J. Appl. Meteor., 9, 514-522.

Leaitch, W. R., G. A. Isaac, J. W. Strapp, C. M. Banic, and H. A. Wiebe, 1992: The relationship between cloud droplet number concentrations and anthropogenic pollution: Observations and climatic implications. J. Geophys. Res., 97, 2463-2474.

Martin, G. M., D. W. Johnson, and A. Spice, 1994: The measurement and parameterization of effective radius of droplets in warm stratocumulus clouds. J. Atmos. Sci., 51, 1823-1842.

Pincus, R., and M. B. Baker, 1994: Effect of precipitation on the albedo susceptibility of clouds in the marine boundary layer. Nature, 372, 250-252.

Platnick, S., and S. Twomey, 1994: Determining the susceptibility of cloud albedo to changes in droplet concentration with the Advanced Very High Resolution Radiometer. J. Appl. Meteor., 33, 334-347.

- and Coauthors, 2000: The role of background cloud microphysics in the radiative formation of ship tracks. J. Atmos. Sci., 57, 2607-2624

Radke, L. F., J. A. Coakley Jr., and M. D. King, 1989: Direct and remote sensing observations of the effects of ships on clouds. Science, 246, 1146-1149.

Rogers, D. P., D. W. Johnson, and C. A. Friehe, 1995: The stable internal boundary layer over a coastal sea. Part I: Airborne measurements of the mean and turbulence structure. J. Atmos. Sci., 52, 667-683.

Saunders, R. W., G. Brogniez, J. C. Guriez, R. Meerkotter, and R. Wendling, 1992: A comparison of measured and modeled broadband fluxes from aircraft data during the ICE' 89 field experiment. J. Atmos. Oceanic Technol. 9, 391-406.

Taylor, J. P., and A. S. Ackerman, 1999: A case study of pronounced perturbations to cloud properties and boundary layer dynamics due to aerosol emissions. Quart. J. Roy. Meteor. Soc., 125, 26432662.

-, M. D. Glew, J. A. Coakley Jr., W. R. Tahnk, S. Platnick, P. V. Hobbs, and R. J. Ferek, 2000: Effects of aerosols on the radiative properties of clouds. J. Atmos. Sci., 57, 2656-2670.

Twomey, S., 1974: Pollution and planetary albedo. Atmos. Environ., 8, 1251-1256.

_ 1991: Aerosols, clouds, and radiation. Atmos. Environ., 25, 2435-2442.

Wallace, J. M., and P. V. Hobbs, 1977: Atmospheric Science: An Introductory Survey. Academic Press, $467 \mathrm{pp}$.

Warren, S. G., C. J. Hahn, J. London, R. M. Chevin, and R. L. Jenne, 1988: Global distribution of total cloud cover and cloud type amounts over the ocean. NCAR/TN 317 + STR, NCAR, Boulder, $\mathrm{CO}, 212 \mathrm{pp}$. 